\title{
Erratum
}

\section{Exclusive semi-leptonic decays of bottom mesons in the spectator quark model}

J. Körner and G.A. Schuler

Z. Phys. C - Particles and Fields 38, 511 (1988)

The last two equations in (2) should read:

$h_{-}^{\mathrm{FQD}}=\left\langle c \downarrow\left|J_{-}\right| b \uparrow\right\rangle=\sqrt{2}\left(\sqrt{Q_{+}}+\sqrt{Q_{-}}\right)$,

$h_{+}^{\mathrm{FQD}}=\left\langle c \uparrow\left|J_{+}\right| b \downarrow\right\rangle=\sqrt{2}\left(\sqrt{Q_{+}}-\sqrt{Q_{-}}\right)$.

The same corrections should be done in the paragraph following (2). Equation (4) should read:

$\frac{1}{2} I h_{0}^{\mathrm{FQD}} \simeq H_{0}^{D} \simeq H_{0}^{D^{*}}$, $\frac{1}{\sqrt{2}} I h_{\mp}^{\mathrm{FQD}} \simeq H_{+}^{D^{*}}$.

The rhs of ( $8 \mathrm{a})$ and $(8 \mathrm{~b})$ should be multiplied by a factor $\frac{1}{2}$. Equations (9), (11), (14), (15) and (16) should be multiplied by $\left|V_{b c}\right|^{2}$. In (17) replace $0\left(\left(M_{1} / M_{2}\right)^{2}\right)$ by $0\left(\left(M_{2} / M_{1}\right)^{2}\right)$.

These typographical errors have no effect on any of the numerical results of the paper. 